\title{
Contributo para a preservação digital do Arquivo Digital da Universidade do Porto
}

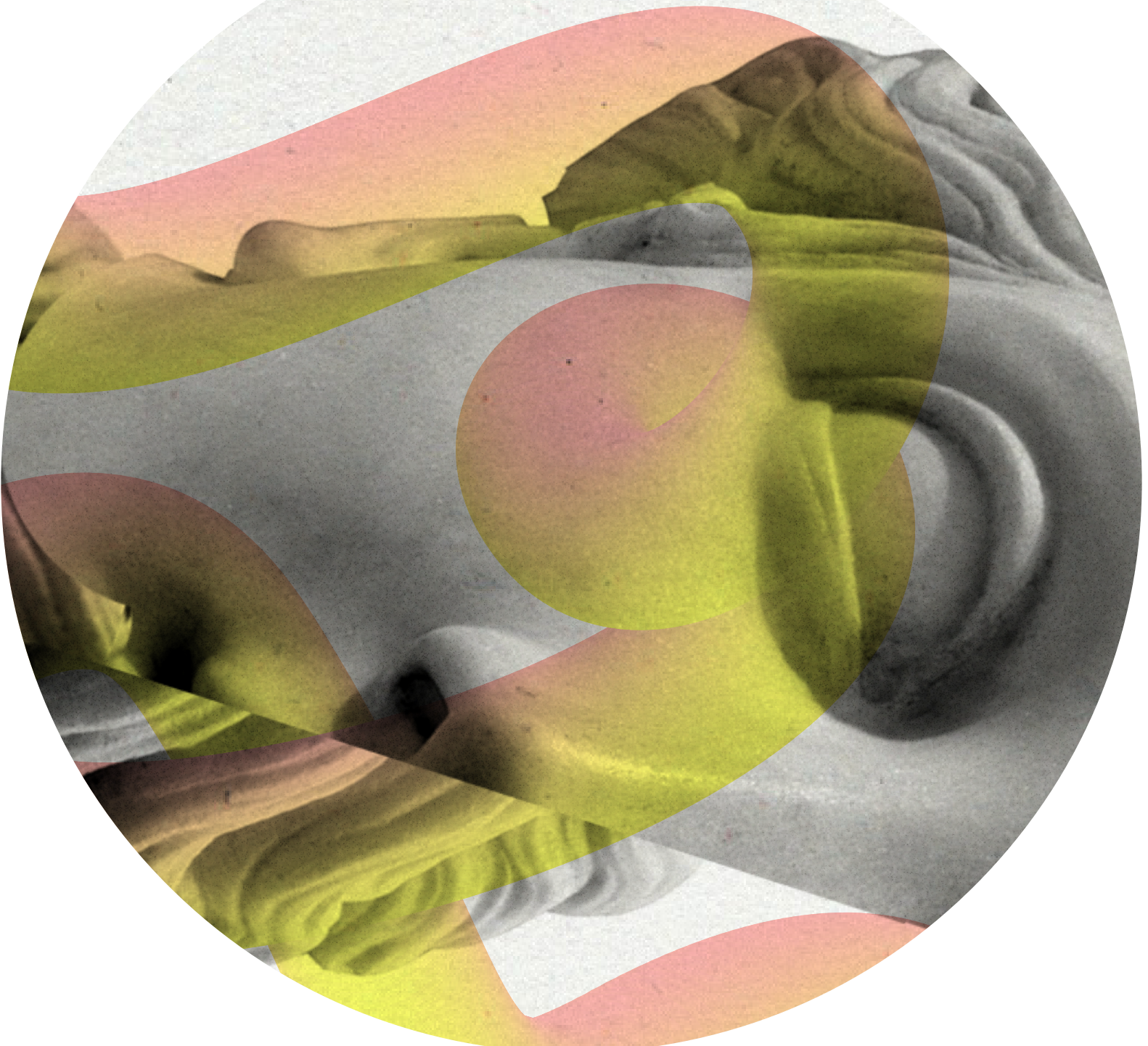

\section{José Miguel Magalhães}

Tavares

jmmta@hotmail.com 


\section{Resumo}

Com este contributo, pretende dar-se a conhecer, de forma sucinta, o trabalho que foi desenvolvido no contexto da componente de aprendizagem baseada no trabalho, em formato de estágio, integrada no curso de especialização do projeto Mu.SA, no âmbito do perfil de Curador de Coleções Digitais. O estágio foi desenvolvido na unidade Gestão de Documentação e Informação da UPDIGITAL, um dos centros funcionais da Reitoria da Universidade do Porto. O objetivo principal consistiu no contributo para a preservação digital de objetos que constituem séries documentais do Arquivo Digital da UPORTO, terminando com a sua inserção numa plataforma específica, o Libsafe. Depois de apresentado o objeto de estudo, os objetivos e a metodologia, passa-se por um sucinto enquadramento sobre temáticas relevantes ao trabalho que foi realizado, como a preservação digital, os suportes, práticas, ferramentas e formatos normalmente utilizados nesta prática. Apresentam-se as séries documentais, as tarefas realizadas e os resultados obtidos, partilhando reflexões e tecendo considerações.

\section{Palavras-chave: Universidade do Porto; Preservação digital; Arquivo digital; Gestão de informação; Tratamento de metainformação}

\section{Abstract}

With this contribution, it is intended to present, in a succinct way, the work that was developed in the context of the work-based learning component, in an internship format, integrated in the specialization course of the Mu.SA project, within the scope of the profile of Digital Collections Curator.

The internship happened in the Documentation and Information Management unit of UPDIGITAL, one of the functional centers of the University of Porto Rectory. The main goal was to contribute to the digital preservation of objects that constitute documental series of the Digital Archive of UPORTO, ending with the insertion of these objects in a specific platform, Libsafe. After the presentation of the object, the objectives and methodology, there is a succinct contextualization of themes that are relevant to the work, such as digital preservation, supports, practices, tools, and formats normally used in this practice. The documental series, the tasks performed, and the results obtained are presented, sharing reflections, and making considerations. 
Keywords: University of Porto; Digital preservation; Digital archive; Information management; Metadata treatment

\section{Nota biográfica}

José Tavares é licenciado em Arqueologia e especialista em Conservação Preventiva de Documentos Gráficos e Fotográficos pela Faculdade de Letras da Universidade do Porto (FLUP). Possui o curso de mestrado em Museologia pela mesma instituição, com principal interesse pela conservação preventiva de coleções. Atualmente, desenvolve trabaIhos em colaboração com uma empresa de conservação e restauro, tendo colaborado com a Oficina de Conservação e Restauro da Biblioteca do Fundo Antigo da Universidade do Porto em tarefas de conservação preventiva, como voluntário.

\section{Biographical note}

José Tavares has a degree in Archaeology and a specialization in Preventive Conservation of Graphic and Photographic Documents from the Faculty of Arts and Humanities of the University of Porto. He also obtained the master's course in Museology from the same institution, with a main interest for the preventive conservation of collections. He currently works with a conservation and restoration company and previously collaborated, as a volunteer, with the Conservation and Restoration Studio of the Old Collection Library of the University of Porto in preventive conservation tasks.

\section{Introdução}

O trabalho que se partilha decorre do estágio de 216 horas realizado na unidade de Gestão da Documentação e Informação do serviço UPDIGITAL, com foco principal no Arquivo Digital. O Arquivo Digital da Universidade do Porto (ADUP):

assegura o acesso agregado a informação produzida e acumulada no contexto sistémico da Universidade, decorrente de atividades levadas a cabo por entidades como os órgãos de governo, a Reitoria, Unidades Orgânicas de Ensino e Investigação e estabelecimentos dependentes, bem como por instituições que antecederam o estabelecimento no Porto do ensino superior universitário. Constituem exemplo 
deste último grupo de instituições a Academia Real de Marinha e Comércio da Cidade do Porto e a Academia Politécnica do Porto (Repositório Temático da Universidade do Porto, (s/da).

Como referido, este arquivo está à responsabilidade da unidade de Gestão da Documentação e Informação (GDI), que se encontra integrada nos Serviços da Informação (SI), parte do centro funcional UPDIGITAL. A plataforma e os conteúdos são alvos de uma gestão centralizada. No entanto, o acesso é distribuído no que toca ao registo e à edição da metainformação e ao depósito de objetos digitais. Este modo de funcionamento é aplicado para permitir a qualidade da informação e a homogeneização dos procedimentos e políticas de arquivo (Repositório Temático da Universidade do Porto, s/da).

Os objetos nado-digitais figuram, cada vez mais, em coleções museológicas. Portanto, torna-se essencial o desenvolvimento de competências de curadoria especializadas neste tipo de objeto. A missão do curador de coleções digitais passa, então, pela implementação de uma estratégia digital que englobe a coleção, armazenamento, preservação e acessibilidade de coleções digitais. Algumas das principais responsabilidades definidas pelo consórcio do Mu.SA para este perfil funcional (Silvaggi \& Pesce, 2017) incluem o contributo para uma melhor preservação digital de um museu, bem como a produção de metainformação relativa aos objetos digitais, assente também na colaboração com vários departamentos do museu. Foi orientado por estas responsabilidades que o estágio se desenvolveu.

\section{Objeto de estudo, objetivos e metodologia 1.1. O objeto de estudo}

O objeto, ou os objetos, de estudo deste trabalho foram definidos previamente pelos responsáveis da unidade, tendo em conta os projetos que estavam a ser desenvolvidos aquando da demonstração de interesse em desenvolver o estágio neste contexto.

Assim, foram objeto de análise a série Recortes de Imprensa [1919-1987], a série Projetos de Instalações [1927-2002] e o Arquivo da Academia Politécnica do Porto (ADUP APP).

A série Recortes de Imprensa [1919-1987] está integrada na subcomunidade da Reitoria da Universidade do Porto (ADUP REIT). Esta série é constituída por notícias publicadas na imprensa, que abordam principalmente assuntos relacionados com a Universidade 
do Porto e o Ensino Superior no geral. Esta série tem vindo a ser intervencionada desde 2008, quando se começou a produzir metainformação relacionada com cada notícia, tendo já sido digitalizados mais de 5000 registos (Repositório Temático da Universidade do Porto, s/dd).

A série Projetos de Instalações [1927-2002] encontra-se também integrada na subcomunidade ADUP REIT. Esta série é constituída por registos que foram resultado de uma transferência de suporte iniciada em 2005, financiada pelo Fundo Europeu de Desenvolvimento Regional, através do Ministério da Cultura. Estas digitalizações foram realizadas no âmbito de um projeto intitulado «A Universidade e a Cidade: Os edifícios da Universidade do Porto ao longo da história - um projeto de tratamento arquivístico e digitalização», e neste foram digitalizados por volta de 10000 desenhos de projeto, e um milhar de processos de obras relativos às instalações da Universidade do Porto (Repositório Temático da Universidade do Porto, s/dc).

O Arquivo da Academia Politécnica do Porto (ADUP APP) divide-se em diversas subcomunidades e relaciona-se principalmente com a documentação produzida pela Academia Politécnica e recuperada pela Reitoria da Universidade do Porto. Esta instituição nasceu fruto da reforma total, a 13 de janeiro de 1837, da Academia Real de Marinha e Comércio da Cidade do Porto, fundada em 1803 (Repositório Temático da Universidade do Porto, s/db). A Academia Politécnica tinha como objetivo principal o ensino das ciências industriais e a formação de engenheiros de várias valências, comerciantes, pilotos e agricultores. Foi inaugurada a 5 de março, com João Baptista Ribeiro (1790-1868) como primeiro diretor, e desmembrada em 1911, sendo integrada parcialmente na nova Faculdade de Ciências. Em 1915, as cadeiras dos cursos de Engenharia passam a constituir a Faculdade Técnica, que em 1926 dá origem à Faculdade de Engenharia (Repositório Temático da Universidade do Porto, s/db).

\subsection{Os objetivos}

O objetivo principal apresentado no plano de estágio prendeu-se, principalmente, com a preservação digital de objetos constituintes de várias séries documentais do ADUP, de forma a inseri-los na plataforma de preservação digital Libsafe. Essas séries documentais referem-se ao Arquivo da Academia Politécnica do Porto, aos Recortes de Imprensa do Arquivo da Reitoria e aos Projetos de Instalações do Arquivo da Reitoria.

De modo a atingir este objetivo, foi necessário o cumprimento de determinadas tarefas, 
sendo de destacar: a verificação, edição e validação da metainformação dos objetos digitais, através da leitura em tabelas abertas em LibreOffice Calc e Microsoft Excel; a caraterização e validação dos mesmos através de ferramentas de acesso aberto, nomeadamente DROID, JHOVE e EXIFTOOL; a criação dos Submission Information Packages, através da ferramenta de acesso aberto SAFBuilder, para a submissão na plataforma.

Estes objetivos foram definidos em função dos problemas e necessidades do ADUP. Os principais problemas verificaram-se nas bases de dados de metainformação dos objetos digitais, onde existem colunas repetidas ou obsoletas, e valores divididos entre várias colunas iguais, bem como alguns problemas relacionados com o repositório temático do ADUP, onde algumas imagens não estavam presentes, provavelmente devido a erros internos aquando da importação dos PNG dos objetos digitais.

\subsection{A metodología}

De modo a atingir os objetivos, começou por se realizar uma breve revisão da literatura sobre preservação digital e de todas as práticas que lhe estão associadas, em paralelo com o trabalho que ia sendo realizado.

Foram realizadas pequenas sessões de formação à medida que o trabalho ia avançando, com o objetivo de um desenvolvimento conciso e informado das tarefas. Para além dos momentos de formação mais formais, as conversas de caráter mais casual também foram instrumentos úteis de recolha de informação e de conhecimentos, através dos vários técnicos que trabalham dentro e fora da área, na mesma unidade.

\section{Resultados e discussão \\ 2.1. A preservação digital}

A preservação digital, como afirma Rua (2016), surge como resposta à possível perda de património existente em meio digital e ao risco permanente da obsolescência das tecnologias e dos formatos. Ferreira (2006) define este mesmo termo como

(...) o conjunto de atividades ou processos responsáveis por garantir o acesso continuado a longo-prazo à informação e restante património cultural existente em formatos digitais (p. 20).

A UNESCO define a preservação digital da seguinte forma: 
The processes aimed at ensuring the continued accessibility of digital materials. To do this involves finding ways to re-present what was originally presented to users by a combination of software and hardware tools acting on data. To achieve this requires digital objects to be understood and managed at four levels: as physical phenomena; as logical encodings; as conceptual objects that have meaning to humans; and as sets of essential elements that must be preserved in order to offer future users the essence of the object (UNESCO, s/d).

Em sintonia com Ferreira (2006), considera-se que a preservação digital é um tema relativamente recente, vasto e complexo. Isto porque só há cerca de uma década é que se começou a afirmar, possuindo ainda vários problemas e questões conceptuais, práticas e tecnológicas. A informação digital é algo crescente em termos de volume, possuindo caraterísticas diversas, com facilidade na sua manipulação, interligação e reutilização, o que torna este campo ainda mais apelativo. $O$ objetivo principal da preservação digital, nas palavras de Santos e Flores (2015), consiste na "atividade de garantir o acesso à informação em meio digital, mantendo a sua integridade e autenticidade"' (p. 90).

Desde que se produz documentação escrita que há uma preocupação em preservar os artefactos resultantes deste e de vários outros processos intelectuais e criativos. Esta preservação tem, claro, o objetivo de permitir a compreensão e contextualização histórica e cultural por parte das gerações futuras (Ferreira, 2006). Estes artefactos físicos são, como se sabe, integrados na missão dos museus, arquivos e bibliotecas, que pretendem preservar estes objetos ou documentos. Contudo, na contemporaneidade existem cada vez mais produções que nascem já em ambiente digital, o que as torna dependentes dos contextos tecnológicos, tornando-as particularmente vulneráveis à obsolescência, numa conjuntura mundial em que a tecnologia avança a largos passos e em curtos períodos de tempo.

\subsubsection{Os suportes de preservação digital}

Quanto aos suportes de preservação digital, existem vários que foram utilizados ao longo do tempo. No entanto, atualmente, sabe-se que a maioria destes possui problemas a nível de preservação, para além de estarem também obsoletos a vários níveis. Podem referir-se as cassetes Betamax e VHS. As primeiras surgem em 1975 e são descontinuadas em 2016 (Kleinman, 2015). As segundas surgem em 1976 e a sua produção termina 
em 2016, no Japão, mas em 2008 nos Estados Unidos da América (Boucher, 2008).

As cassetes VHS foram, de forma geral, mais populares do que as Betamax, visto que a Sony não facilitava, segundo Ferreira (2006), o licenciamento de produção a terceiros. Atualmente é muito difícil encontrar um dispositivo que consiga ler cassetes Betamax. Quanto ao VHS, ainda existem alguns leitores, mas, ainda assim, começam a escassear ou possuem preços altos pelo seu valor "vintage". Estes suportes são bons exemplos do que acontece com a maioria dos suportes produzidos atualmente, ou seja, a tecnologia evolui de forma tão exponencial que acaba por aumentar o nível e a probabilidade de obsolescência dos suportes.

Outros exemplos contam com as disquetes de 3.5 polegadas, extremamente populares e utilizadas inclusive em contextos escolares. Entretanto, deixaram de ser utilizadas e, em 2003, os computadores Dell já não possuíam leitores integrados deste tipo de suporte. Os restantes fabricantes rapidamente os seguiram (Ferreira, 2006). Depois das disquetes, surgiram outros suportes como os DVD e as flash-drives, bem como os discos externos, que são atualmente os mais utilizados. No entanto, sabe-se que todos estes suportes possuem problemas, devido principalmente ao seu tempo de vida limitado. Daí, a importância da preservação digital, para se evitar a perda de quantidades de informação avultadas, o que já tem vindo a acontecer ao longo do tempo.

\subsubsection{As práticas, estratégias e políticas}

A preservação digital é, portanto, um processo vasto e com uma componente técnica bem presente. No entanto, existem hoje em dia algumas práticas e estratégias de preservação digital já documentadas e mencionadas por norma nas plataformas e publicações científicas relacionadas com este tema. A preservação digital compreende, como afirmam Santos e Flores (2015), procedimentos de ordem estrutural e operacional, sendo que os primeiros são os "investimentos iniciais" (p. 90) enquanto os segundos são os aplicados para a preservação física, lógica e intelectual dos documentos digitais. Os procedimentos estruturais são o equivalente das políticas de preservação e os operacionais são as atividades técnicas realizadas sob os documentos digitais. As estratégias usam, como foco, os vários níveis do objeto digital, sendo esses o físico, o lógico e o conceptual. Estes níveis são bem definidos por Thibodeau (2002), afirmando que o objeto físico é o que é interpretado pelo hardware, transformado posteriormente em objeto lógico, que é interpretado pelo software, que o transforma em objeto conceitual, o nível interpretável pelo ser humano. 
As estratégias de preservação digital estão bem documentadas num relativamente grande volume de obras, mas destacam-se, neste trabalho, os estudos de Ferreira (2006) e de Rua (2016). Apresentam-se, de forma relativamente sucinta:

- A migração para suportes analógicos, que consiste na reprodução de um objeto digital em papel ou qualquer outro suporte analógico;

- A preservação de tecnologia, que integra a manutenção do hardware e software necessários para aceder ao objeto. É uma das estratégias mais importantes e difíceis, devido à obsolescência tecnológica;

- O refrescamento de suporte, que consiste na atualização do suporte físico onde o objeto se encontra armazenado, seja esse um disco rígido, um DVD ou outro;

- A emulação, ou recriação das condições de acesso ao objeto recorrendo a tecnologias recentes. É uma estratégia que se concentra na autenticidade dos objetos digitais e na manutenção da sua funcionalidade. É usada, por exemplo, para executar num computador jogos de sistemas antigos;

- A migração é o transporte de recursos digitais de uma plataforma tecnológica para outra, geralmente realizada quando a plataforma original está em risco de obsolescência ou já com outros problemas tecnológicos. No entanto, esta estratégia pode comprometer a autenticidade do objeto devido à necessidade de rescrição de parte do mesmo;

- O XML pode ser visto como uma migração particular. Sendo uma "linguagem de enriquecimento de informação sobre estruturas e significado" (Rua, 2016, pp. 34-35), independente da plataforma, é favorecedor da interoperabilidade;

- O encapsulamento engloba o objeto no seu formato original em conjunto com metainformação descritiva do formato do ficheiro, que leva à interpretação do original quando é necessária a utilização de conversores ou emuladores;

- A Pedra de Roseta digital é um último recurso utilizado quando não existe informação suficiente acerca do formato do objeto digital. Funciona através de amostras representativas do mesmo, que "deverão existir num formato diretamente interpretado pelo ser humano que deles inferirá as regras" (Rua, 2016, p. 44).

As políticas de preservação digital possuem, por norma, alguns pontos em comum. Isto é, as políticas que realmente se aplicam à preservação digital na sua aceção real, e não 
simplesmente associada à digitalização, com a qual foi confundida durante algum tempo. Geralmente, as políticas de preservação digital referem-se ao conceito e aos desafios, à necessidade de adoção de normas abertas e ao uso do modelo concetual Open Archival Information System (OAIS), e a existência de documentos que guiem o processo de digitalização (Rua, 2016). No que toca a este tipo de políticas, o National Museum, o National Archive e a National Library of Australia são instituições de referência no contexto da preservação digital. Destas políticas, salientam-se alguns pontos essenciais:

- O uso do modelo OAIS e da norma de metainformação Australian Government Locator Service (AGLS);

- A importância da interoperabilidade para sistemas externos;

- O uso de normas abertas para a digitalização e a sua revisão regular;

- Menção às estratégias de preservação digital utilizadas;

- Priorização da preservação de documentos ou objetos que estejam em risco de perda de informação;

- A não-alteração de imagens originais ou derivadas sem autorização devida (National Museum of Australia, 2012; National Library of Australia, 2013).

O modelo OAIS surgiu a partir de 1990, quando a Consultative Committee for Space Data Systems (CCSDS) e a International Organization for Standardization (ISO) realizam um esforço conjunto para desenvolver normas "capazes de regular o armazenamento a longo prazo de informação digital produzida no âmbito de missões espaciais" (Ferreira, 2006, pp. 27-31). Deste esforço surge um modelo concetual que visa identificar os componentes funcionais que deverão fazer parte de um sistema de informação dedicado à preservação digital, descrevendo também interfaces internas e externas do mesmo sistema e os objetos que estão no seu interior. Foi aprovado como norma internacional em 2003, a norma ISO Standard 1471:2003. Este modelo permitiu, primeiro que tudo, que existisse uma normalização na linguagem dos profissionais que trabalham nesta área.

Como explica Ferreira (2006), o produtor é a entidade que submete o material ao repositório ou plataforma. Este material submetido é o Submission Information Package (SIP ou PIS). Durante este processo de submissão, denominado ingestão, o repositório tem de garantir a integridade da informação recebida, e aqui é produzida a informação descritiva, a metainformação, que é gerida pela Gestão de Dados, e o material a preservar, 
agora transformado no Archival Information Package (AIP ou PIA), é conservado no Repositório de Dados. O pacote que é entregue ao consumidor, ou seja, a comunidade interessada na informação, é denominado por Dissemination Information Package (DIP ou PID). A Administração é a responsável pelas operações de manutenção e monitorização dos processos decorridos no repositório. Na Fig. 1, é possível observar o esquema de todo o processo.

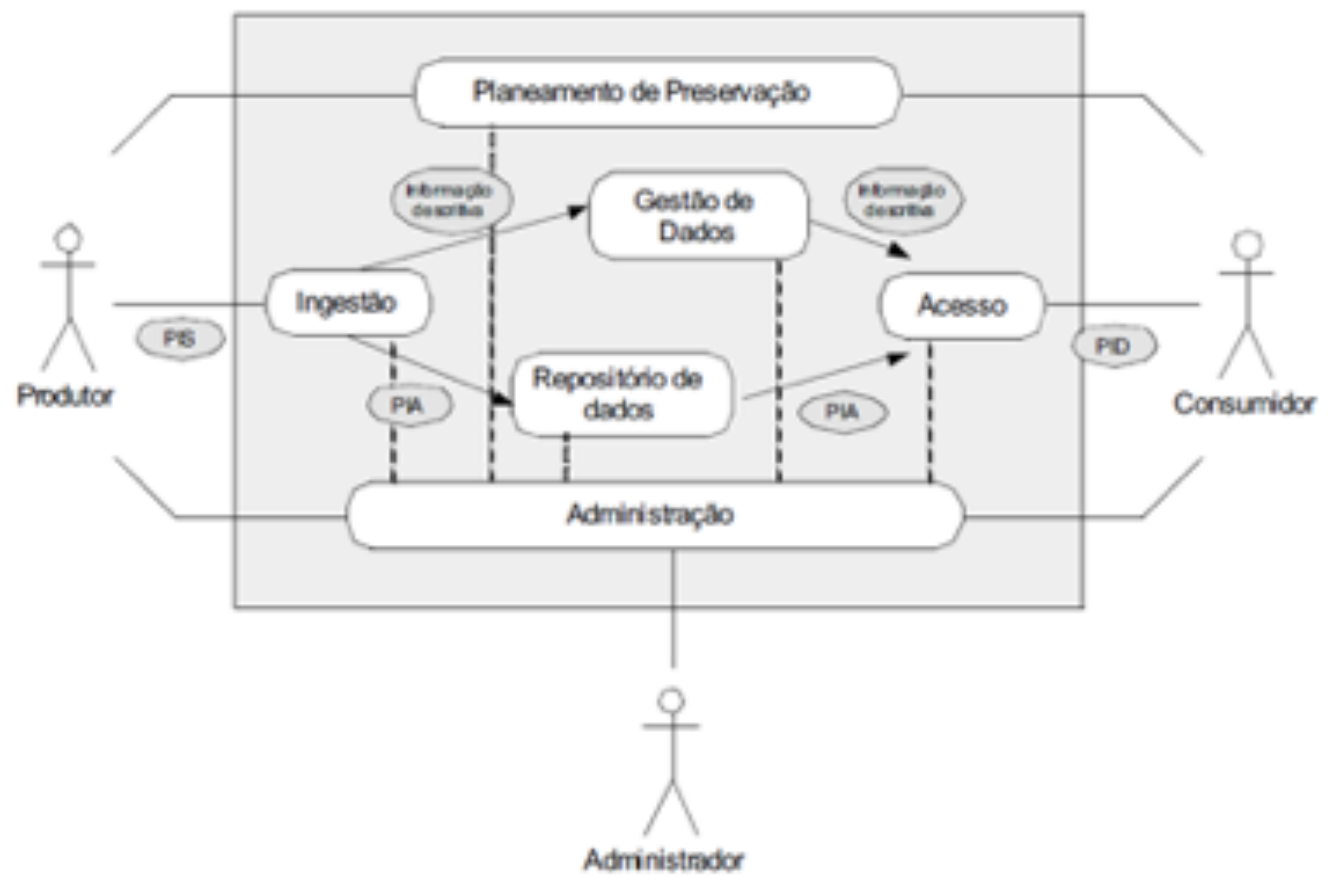

Fig. 1 - O modelo OAIS (Ferreira, 2006, p. 28)

\subsubsection{Formatos e ferramentas}

Normalmente, os formatos principais de preservação digital são o TIFF e o PDF/A.

Tal acontece no ADUP. Existem outros formatos que podem ser utilizados como 0 JPEG2000. No entanto, este não é utilizado no contexto do ADUP.

No que diz respeito às ferramentas utilizadas na preservação digital, destacam-se as que foram utilizadas no estágio, de acesso aberto:

- DROID, uma ferramenta de identificação de formatos de ficheiro, desenvolvida pela The National Archives com o objetivo de identificar formatos em lote (The National Archives, $\mathrm{s} / \mathrm{d})$;

- JHOVE, ferramenta de identificação, validação e caraterização de formatos, criada em colaboração pela Journal Storage (JSTOR) e pela Universidade de Harvard com recurso a 
Java (Open Preservation Foundation, s/d);

- ExifTool, uma ferramenta de leitura, escrita e edição de metainformação que permite uma variedade de formatos, podendo também ser utilizada para a caraterização de formatos;

- SafBuilder, uma ferramenta que transforma ficheiros de conteúdo e metainformação num pacote Simple Archive Format, possibilitando a importação em lote para plataformas como o DSpace, sendo utilizado com recurso a Java.

\subsection{Preservação digital no Arquivo Digital da Universidade do Porto 2.2.1. Introdução às atividades e testagem}

Inicialmente, as atividades realizadas na instituição focaram-se sobretudo na pesquisa bibliográfica e na introdução a noções básicas acerca do trabalho desenvolvido e a desenvolver. Isto porque foi necessário investir previamente na formação geral complementar, visto que não foram dadas competências específicas de preservação digital nos restantes módulos do curso de especialização. Foi realizada uma visita guiada ao depósito e ao local de trabalho geral, bem como aos espaços de acondicionamento e unidades de controlo ambiental que estão instalados no depósito. Foram apresentados os suportes físicos para os objetos digitais e as suas desvantagens e vantagens, sendo o foco a obsolescência tecnológica destes suportes e a necessidade de migração ou refrescamento.

Foram sendo dados vários enquadramentos teóricos relacionados com a preservação digital ao longo do estágio, de modo a afinar os temas, conceitos e práticas. Para além disto, foram sendo realizados vários testes relacionados com o uso das ferramentas de acesso aberto já referidas para caraterização e validação, a digitalização e os formatos utilizados. Foram realizados, com recurso ao SAFBuildere ao Rodaln, testes de criação de SIP e testes de validação, identificação e caraterização com recurso ao DROID, JHOVE, PDFINFO e EXIFTOOL.

Foram também referidos alguns conceitos e termos que, apesar de não serem utilizados de forma regular no trabalho a desenvolver no estágio, são importantes e de interesse para o trabalho na área dos arquivos e bibliotecas. Por exemplo, foram referidos termos como o Reconhecimento Ótico de Carateres, ou OCR, as diferenças entre a importação e a importação migração no contexto do DSpace, em que a principal diferença é a presença de um ficheiro handle na segunda opção, e a interoperabilidade de sistemas, que 
consiste principalmente na recolha de informação numa plataforma por outra, ou a ligação entre vários sistemas no que toca à informação. Como exemplo, mencionaram-se as plataformas do Registo Nacional de Teses e Dissertações (RENATES) e dos Repositórios Científicos de Acesso Aberto de Portugal (RCAAP). Para além disto, foram mencionados os tipos de ficheiros usados na criação dos SIP, nomeadamente, os ficheiros baggit, que podem possuir checksums de formato Md5 ou Sha256, com níveis de segurança distintos, por exemplo, o Sha256 possui uma hash única para cada ficheiro, permitindo verificar falhas de segurança informática.

\subsubsection{Tratamento de metainformação e digitalização}

Depois da componente mais teórica e relacionada com testes, começou-se a trabalhar no tratamento de metainformação relativa aos ficheiros csv dos 14 conjuntos da série Recortes de Imprensa da Reitoria. Este tratamento consistiu no uso do LibreOffice Calc ou Microsoft Excel para normalizar campos, eliminar colunas repetidas e/ou desnecessárias, de forma manual ou com recurso a funções para unir colunas ou eliminar determinadas porções de texto dentro das células.

Depois do tratamento de metainformação, que foi um processo ainda demorado devido à presença de muitas inconsistências e vários erros, foi feita a extração de metainformação com recurso às ferramentas de identificação e validação já mencionadas. Foi realizada uma digitalização do último conjunto, AN14, em forma de teste para verificar formatos e perfis de digitalização típicos, e utilizaram-se as mesmas ferramentas para validação e identificação, de forma a comparar com a digitalização original.

De seguida, procedeu-se à criação de ficheiros csv com os handles das imagens, obtidos através de um script de Windows Powershell. As tabelas destes ficheiros foram, depois, adicionadas ao restante conteúdo dos ficheiros csv com a restante metainformação, de modo a criar um ficheiro "final". No entanto, primeiro tinham de ser corrigidos alguns erros deixados pelo passado, que contavam principalmente com ficheiros PNG partidos, mas sem uma correspondência direta com o ficheiro TIFF, que correspondia somente a um dos ficheiros e não a todos. Existiam, para além deste tipo de erro, outros como a presença de recortes de imprensa que acabaram por não ser utilizados por terem gralhas, ou outros repetidos. Esta validação de metainformação foi também um processo demorado e, entretanto, foram feitas mais experiências de digitalização de algumas das imagens 
que tinham erros, nomeadamente as imagens do conjunto AN1e AN2.

\subsubsection{Criação de pacotes de informação de submissão}

Depois de todos os ficheiros de metainformação estarem devidamente tratados e corrigidos, era altura de criar os pacotes de informação de submissão para cada um dos conjuntos de recortes. Este processo envolve os seguintes passos:

- Passa-se o ficheiro csv final com toda a metainformação descritiva para a pasta IMAGES onde se encontram todas as imagens, em formato TIFF (Fig. 2);

- Abre-se a ferramenta SAFBuilder através da linha de comandos (cmd) com argumento "-c" e indicação do caminho do ficheiro de metainformação, que leva à criação de um ficheiro nomeado SimpleArchiveFormat (Fig. 3 e 4);

- O pacote está então criado, e contém todos os itens separados em pastas, neste caso, todos os grupos de imagens referentes a um registo, bem como o ficheiro contents e o ficheiro Dublin Core de metainformação (Fig. 5);

- O pacote precisa de ser renomeado e, para isso, utiliza-se um script de Windows Powershell para renomear as pastas "item" para o registo a que se referem e o SimpleArchiveFormat para a coleção referente no repositório, neste caso nomeada "Collection_ REIT_31791" (Fig. 6). 
Fig. 2 - Passagem do ficheiro de metainformação para a pasta IMAGES. ๑José Tavares

Fig. 3 - Uso do

SAFBuilder através da linha de comandos, criação do pacote de submissão de informação. ๑José Tavares 
Fig. 4 - Todas as imagens divididas em pastas, antes da renomeação. ๑ José Tavares

Fig. 5 - O ficheiro dublincore, contents e a imagem TIF no interior de cada pasta "item". @José Tavares

\begin{tabular}{|c|c|c|c|c|c|c|c|c|c|c|}
\hline \multicolumn{8}{|c|}{ 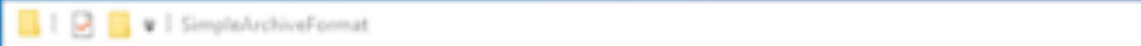 } & \multirow{2}{*}{\multicolumn{3}{|c|}{-8}} \\
\hline Rohero & Dase & Shanar & wer & & & & & & & \\
\hline 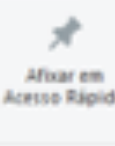 & ide capiar C & & 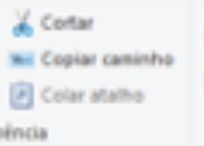 & \multicolumn{2}{|c|}{ 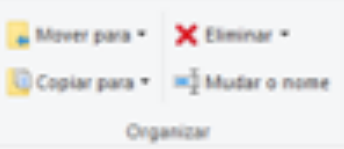 } & $\begin{array}{l}\text { Th. } \\
\text { now tos } \\
\text { nows }\end{array}$ & 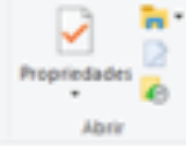 & \multicolumn{3}{|c|}{ 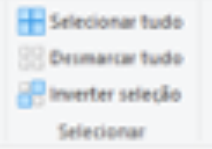 } \\
\hline \multirow[t]{22}{*}{$\leftarrow$} & $*+\square=$ & " Anes & 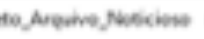 & , Avis , Mus & SES + Simpledeche. & fermat , & 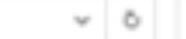 & \multicolumn{3}{|c|}{ P Nocurar ams 5 - } \\
\hline & Afe & A & Neme & A & Dute de m & afficaplo & Tipo & Tamarke & & A \\
\hline & an: & & tem_1 & & t4t20007 & cost & Puts de ficheros & & & \\
\hline & alu & & $\tan 2$ & & 1402008 & Ches & Pable de ficherios & & & \\
\hline & Ates & & $1 \mathrm{~atm}$, & & tusacat & onest & Datue be fisheress & & & \\
\hline & ans & & 11 intun_4 & & 1402008 & 04.49 & Dabs be ficheires & & & \\
\hline & and & & atens & & Hescos & ones & Pats de ficheros & & & \\
\hline & Anat & & $11 \operatorname{sem} 6$ & & 1402003 & 09.49 & Pastes te ficheres & & & \\
\hline & Aten & & $\operatorname{sen} \gamma$ & & Hescax & oseas & Pats Se fichenos & & & \\
\hline & AN? & & $\operatorname{arm} s$ & & Mescos & 969 & Paite de ficheres & & & \\
\hline & Allo & & $1]$ teng & & Hetaser & $08=9$ & Pats de ficherios & & & \\
\hline & AN11 & & Dam,10 & & Hescas & Som & Daits ate fisheres & & & \\
\hline & AN12 & & 11 ten,11 & & 14tedact & 0009 & Pats de ficheings & & & \\
\hline & avis & & $12 \tan , 12$ & & 14tecoar & $\infty=2$ & Bats de ficheros & & & \\
\hline & I deid,jents & & $13 \operatorname{ten}, 13$ & & 14teatex & Oenelp & Puts se ficherios & & & \\
\hline & exifool met & & $10 \mathrm{ntm} / 14$ & & t4tecoos & Otas & Pats de ficheros & & & \\
\hline & masers. & & 11 tem,15 & & 14tescoxt & seas & Puts be ficheros & & & \\
\hline & & & inten_16 & & 14620080 & Ches & Dastu be ficherios & & & \\
\hline & SmpleAse & & 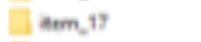 & & twescax & sen & Datu te ficheress & & & \\
\hline & $11 \operatorname{sen} 1$ & & 17 atm_18 & & 14020080 & 04.99 & Pasto de ficheiros & & & \\
\hline & {$[\mathrm{sen} 2$} & & $10 \mathrm{am}, 10$ & & Hesoar & 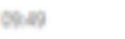 & Pats be ficheress & & & \\
\hline & $1 \mathrm{cam}$ stiec & cients & $110 \mathrm{~atm} 20$ & & 14020020 & 09.49 & Path se ficheries & & & * \\
\hline
\end{tabular}

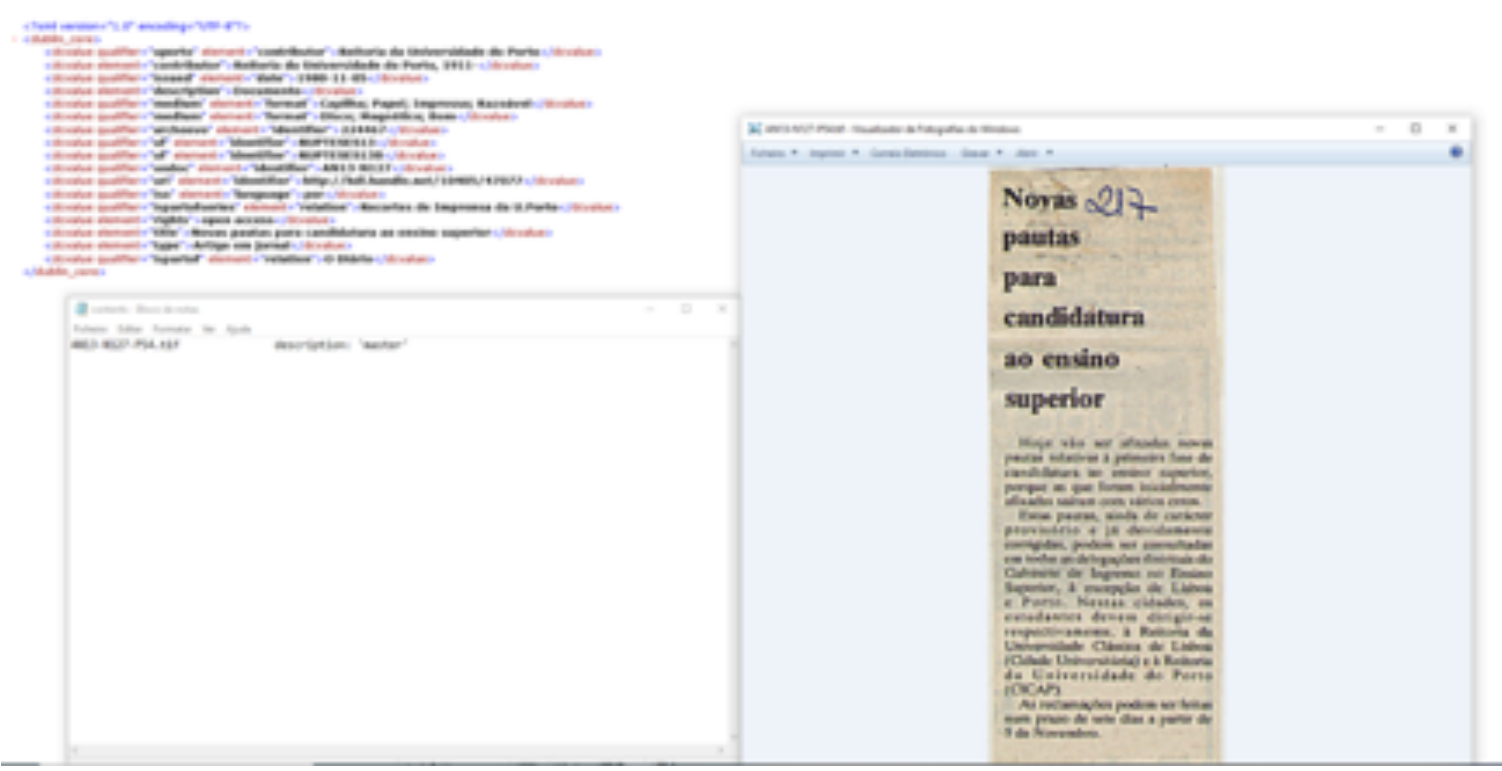


serviço UPDIGITAL, ou seja, foi feita uma integração total no contexto de trabalho que é desenvolvido. Foi possível contactar com diferentes profissionais de diversas áreas, que contribuíram também para a formação de um Curador de Coleções Digitais. Em suma, o trabalho de estágio foi uma experiência que permitiu a obtenção de competências gerais e específicas, bem como o desenvolvimento de capacidades de trabalho de equipa com profissionais de diferentes áreas.

Os objetivos foram cumpridos, tendo sido realizado todo o trabalho que foi delineado na proposta.

\section{Agradecimentos}

O autor agradece à Prof. Doutora Paula Menino Homem, pela oportunidade, à Prof. Doutora Maria Manuela Pinto e ao Dr. João Pereira, pela orientação deste trabalho, pela disponibilidade e pelo conhecimento transmitido e aos restantes membros da unidade GDI, pelo acolhimento.

\section{Referências}

Boucher, G. (2008, dezembro 22). VHS Era is Winding Down. Los Angeles Times. https:// www.latimes.com/archives/la-xpm-2008-dec-22-et-vhs-tapes22-story.html

Ferreira, M. (2006). Introdução à Preservação Digital - Conceitos, Estratégias e Actuais Consensos. Guimarães: Escola de Engenharia da Universidade do Minho.

Kleinman, Z. (2015, novembro 10). Sony Says Goodbye to Betamax Tapes. BBC News. https://www.bbc.com/news/technology-34776424

National Museum of Australia. (2012). Digital Preservation and Digitisation Policy. https://www.nma.gov.au/about/corporate/plans-policies/policies/digital-preservation-and-digitisation

National Library of Australia. (2013). Digital Preservation Policy. $4^{\text {th }}$ Edition. https://www. nla.gov.au/policy-and-planning/digital-preservation-policy

Open Preservation Foundation. (s/d). Getting Started with JHOVE. https://jhove.openpreservation.org/getting-started/ 
O'Flaherty, E. (2018). Digital preservation for libraries, archives, and museums. Archives and Records, 39, 94-97.

Pinto, M. M. G. A. (2009). Gestão de Informação e Preservação Digital: uma perspetiva portuguesa de uma mudança de paradigma. In Congreso del Capítulo Español de ISKO, 9ํ, Valência, 2009 - Nuevas Perspectivas para la Difusión y Organización del Conocimiento: Actas del Congresso (pp. 323-355). València: Universitat Politècnica de València. https://hdl.handle.net/10216/25380

Repositório Temático da Universidade do Porto. (s/da). ADUP - Arquivo Digital da U.Porto. https://repositorio-tematico.up.pt/handle/10405/2084?locale=pt

Repositório Temático da Universidade do Porto. (s/db). ADUP APP - Academia Politécnica do Porto, 1837-1911. https://repositorio-tematico.up.pt/handle/10405/31763

Repositório Temático da Universidade do Porto. (s/dc). ADUP REIT - Projectos de instalações [1927-2002]. https://repositorio-tematico.up.pt/handle/10405/31790

Repositório Temático da Universidade do Porto. (s/dd). ADUP REIT - Recortes de imprensa [1919-1987]. https://repositorio-tematico.up.pt/handle/10405/31791

Rua, J. P. A. (2016). Digitalização, Preservação Digital e Acesso em Instituições de Memória - Contributos para o Projeto Museu Digital da U.Porto. (Dissertação de Mestrado, Universidade do Porto).

Santos, H., \& Flores, D. (2015). Estratégias de preservação digital para documentos arquivísticos: uma breve reflexão. Cadernos BAD, 1, 87-101.

Silvaggi, A., \& Pesce, F. (Eds.). (2017). Museum Professionals in the Digital Era, Agents of Change and Innovation. Bolonha: Melting Pro \& Mu.Sa - Museum Sector Alliance. http:/ / www.project-musa.eu/wp-content/uploads/2020/06/MuSA-Emerging-Job-Profiles-for-museum-professionals.pdf

The National Archives. (s/d). Download DROID: File Format Identification Tool. https:// www.nationalarchives.gov.uk/information-management/manage-information/preserving-digital-records/droid/

Thibodeau, K. (2002). Overview of technological approaches to digital preservation and challenges in coming years. The State of Digital Preservation: An International Perspective. CONFERENCE PROCEEDINGS, 4-31. https://chnm.gmu.edu/digitalhistory/links/ 
pdf/preserving/8_37e.pdf

UNESCO. (2009). Charter on the Preservation of the Digital Heritage. https://unesdoc. unesco.org/ark:/48223/pf0000179529.page=2

UNESCO. (s/d). Concept of Digital Preservation. https://en.unesco.org/themes/information-preservation/digital-heritage/concept-digital-preservation

Zhou, M., Geng, G., \& Wu, Z. (2012). Digital Preservation Technology for Cultural Heritage. Higher Education Press. 
